\title{
Phase Locking, Period Doubling Bifurcations and Chaos in a Mathematical Model of a Periodically Driven Oscillator: A Theory for the Entrainment of Biological Oscillators and the Generation of Cardiac Dysrhythmias
}

\author{
Michael R. Guevara and Leon Glass \\ Department of Physiology, 3655 Drummond Street, McGill University, Montreal, Quebec H3G 1Y6, \\ Canada
}

\begin{abstract}
A mathematical model for the perturbation of a biological oscillator by single and periodic impulses is analyzed. In response to a single stimulus the phase of the oscillator is changed. If the new phase following a stimulus is plotted against the old phase the resulting curve is called the phase transition curve or PTC (Pavlidis, 1973). There are two qualitatively different types of phase resetting. Using the terminology of Winfree (1977, 1980), large perturbations give a type 0 PTC (average slope of the PTC equals zero), whereas small perturbations give a type 1 PTC. The effects of periodic inputs can be analyzed by using the PTC to construct the Poincare or phase advance map. Over a limited range of stimulation frequency and amplitude, the Poincaré map can be reduced to an interval map possessing a single maximum. Over this range there are period doubling bifurcations as well as chaotic dynamics. Numerical and analytical studies of the Poincare map show that both phase locked and non-phase locked dynamics occur. We propose that cardiac dysrhythmias may arise from desynchronization of two or more spontaneously oscillating regions of the heart. This hypothesis serves to account for the various forms of atrioventricular (AV) block clinically observed. In particular $2: 2$ and $4: 2 \mathrm{AV}$ block can arise by period doubling bifurcations, and intermittent or variable $\mathrm{AV}$ block may be due to the complex irregular behavior associated with chaotic dynamics.
\end{abstract}

Key words: Cardiac dysrhythmias - Phase locking - Chaos - Period doubling bifurcations

\section{Introduction}

The rhythm of autonomous biological oscillators can be markedly affected by periodic perturbation. Studies of the effects of periodic electrical stimulation of neural and cardiac oscillators have revealed that the intrinsic rhythm may become entrained or phase locked to the periodic stimulus (Perkel et al., 1964; 
Moulopoulos et al., 1965; Reid, 1969; van der Tweel et al., 1973; Ayers and Selverston, 1977; Pinsker, 1977; Jalife and Moe, 1979; Scott, 1979; Guttman et al., 1980; Guevara et al., 1981). Phase locking in an $N: M$ ratio occurs when there are $M$ cycles of the driven oscillator for every $N$ stimuli. In $N: M$ phase locking one observes $M$ marker events associated with the driven cycle (for example, upstrokes of the action potential) occurring at $M$ different times or phases in the stimulus cycle. The $N: M$ pattern is a repeating sequence periodic in time.

In addition to phase locked dynamics, irregular or aperiodic dynamics have been observed in theoretical studies (Cartwright and Littlewood, 1945; Levinson, 1949; Zaslavsky, 1978; Tomita and Kai, 1978; Guckenheimer, 1980; Levi, 1981). The term "chaos" has been adopted by recent workers ( $\mathrm{Li}$ and Yorke, 1975; May, 1976) to describe complex and aperiodic dynamics that can arise in deterministic systems (i.e. systems described by equations that do not include stochastic terms). Experimentalists have often observed dynamics which were not phase locked in response to periodic stimulation of biological oscillators (Perkel et al., 1964; Moulopoulos et al., 1965; Reid, 1969; van der Tweel et al., 1973; Ayers and Selverston, 1977; Pinsker, 1977; Ushiyama and Brooks, 1977; Jalife and Moe, 1979; Scott, 1979; Guttman et al., 1980; Petrillo, 1981; Guevara et al., 1981). The usual explanation for such irregular behaviour is that "noise" present in a complex system will tend to destroy periodic patterns (Guttman et al., 1980; Glass et al., 1980). An alternative explanation is that at least some of the irregular behaviour is a reflection of chaotic dynamics intrinsic to the deterministic equations describing the system.

In principle, numerical computation of the effects of periodic input on equations displaying limit cycle behaviour should be a straightforward procedure. However, since realistic models of biological oscillators are formulated as stiff systems of several nonlinear differential equations (Hodgkin and Huxley, 1952; McAllister et al., 1975), numerical integration of such equations in response to periodic input is an expensive and time consuming procedure and has seldom been carried out (Holden, 1976; Guttman et al., 1980). In addition, it is difficult, even with simplified systems such as the van der Pol oscillator, to obtain a clear view of the dynamics in response to periodic stimulation (Cartwright and Littlewood, 1945; Levinson, 1949; Flaherty and Hoppensteadt, 1978; Scott, 1979; Guckenheimer, 1980; Levi, 1981). Therefore, in order to investigate the effect of periodic stimulation, we have developed a general mathematical model that is simple enough to allow use of an alternative approach to numerical integration of nonlinear differential equations.

If a biological oscillator is subjected to a single, short (in comparison to its period of oscillation) stimulus, its rhythm undergoes a transient disturbance. Following the transient period, the oscillation eventually reverts to its original rate, but with a resetting of the phase of the oscillation (Winfree, 1977). We obtain an analytic expression for the response of our simple two dimensional oscillator to such an isolated stimulus (the phase transition curve or PTC). Using the analytic form of the PTC, we then investigate the response of the model to periodic stimulation by constructing the Poincare map. This approach of using the response to a single stimulus to predict the response to repetitive stimulation has been previously used (Perkel et al., 1964; Moe et al., 1977; Pinsker, 1977; Scott, 1979). 
There are correspondences between the behaviour displayed by the model and experimental observations, both in regard to the response to a single stimulus and in regard to the response to periodic stimulation. When a periodic stimulus is applied, the model displays phase locked patterns as well as irregular, non-phase locked behaviour that arises out of chaotic dynamics.

Since our work may be of interest to experimentalists, we have written this paper so that the results can be appreciated without understanding the technical details. Section II is a general presentation of the mathematical model and the principal results. Sections III, IV and V are technical and directed towards theoreticians and show how the results were obtained. Section VI is a non-technical discussion of the relevance of this theoretical work to experimental and clinical observations. The Appendices contain proofs and computations.

\section{The Mathematical Model and an Overview of the Results}

In this section we describe the mathematical model and its limitations, and the response of the model to a single isolated stimulus. Next we illustrate the different types of phase locked and irregular dynamics that can result from periodic stimulation.

The mathematical model for the biological oscillator in this paper has previously been discussed by Winfree $(1975,1980)$ and Scott $(1979)$. The model is given in two dimensional polar coordinates by the equations

$$
\begin{aligned}
& \frac{d \Phi}{d t}=2 \pi, \\
& \frac{d r}{d t}=\operatorname{ar}(1-r),
\end{aligned}
$$

where $\Phi$ is the angular coordinate $(-\infty<\Phi<\infty), r$ is the radial coordinate and $a$ is a positive real number. The unit circle forms a limit cycle that is globally attracting for all initial conditions except for the equilibrium point at the origin. Note that the unperturbed oscillator has unit period and its state can be parametrized by an angular coordinate $\phi$ :

$$
\phi=\frac{\Phi}{2 \pi}(\bmod 1) .
$$

The variable $\phi$ is called the phase of the oscillation $(0 \leqslant \phi<1)$.

Perturbation away from the limit cycle results in relaxation back to the stable limit cycle at a rate that depends on the parameter $a$. We consider the limiting case of $a \rightarrow \infty$. In this case, following perturbation away from the limit cycle, there is an instantaneous relaxation back to the limit cycle along a radial direction. In what follows we shall consider perturbations consisting of impulses of magnitude $b$ which are directed parallel to the $x$-axis (Fig. 1). Thus, the effect of a single impulse is to instantaneously reset the phase of the oscillator. Calling $\phi$ the old phase of the oscillation immediately preceeding the perturbation, and $\theta$ the new phase of the oscillation immediately following the perturbation we have

$$
\theta=f(\phi, b),
$$




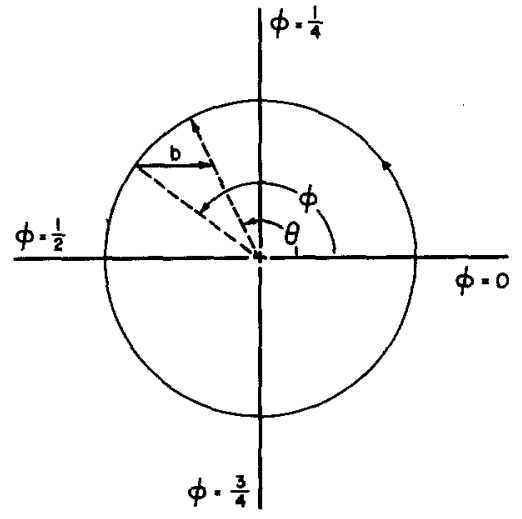

Fig. 1. Schematic representation of the effect of perturbation with an impulse of magnitude $b$ on the model oscillator. The unit circle forms a limit cycle which is globally attracting for all points except the origin. The perturbation instantaneously resets the phase of the oscillator from phase $\phi$ prior to the perturbation to phase $\theta$ after the perturbation. Every time the oscillator passes through phase $\phi=0$, we associate this with an observable event such as a neural or cardiac action potential. Identifying the $x$ axis variable with membrane potential, perturbations directed along the $x$-axis $(b>0)$ are analogous to depolarizations, whereas perturbations directed in the opposite direction $(b<0)$ are analogous to hyperpolarizations

where the function $f$ is called the phase transition curve (PTC). In Section III of this paper we analytically compute the PTC for this model and discuss its properties. Our choice of the model in Fig. 1 has been motivated by the fact that an analytic expression can be found for the PTC. This is in sharp contrast to other simple models of limit cycle oscillations such as the van der Pol oscillator for which analytical expressions for the PTC are not available (Pavlidis, 1973; Winfree, 1980).

In addition to its analytic simplicity, the PTC of (1) displays certain qualitative features which resemble some experimental observations. Consider a perturbation directed along the positive $x$-axis $(b>0)$. Applying the perturbation during the first half of the cycle $(0<\phi<0.5)$ leads to a delay in the phase of the oscillation, whereas application of the same perturbation in the second half of the cycle $(0.5<\phi<1.0)$ leads to an advance of phase. Also, for this model, the average slope of the PTC is 1 (type 1 PTC) at low amplitudes of perturbation and 0 (type 0 PTC) at higher amplitudes. Winfree $(1977,1980)$ reviewed data resulting from different experimental preparations and showed that in many situations the PTC's are biphasic and either type 1 or type 0 .

Despite these parallels with experiment, the mathematical model is unrealistic for many reasons. Since most realistic mathematical models for biological oscillations are systems of differential equations of dimension greater than 2 , the topological dimension of the proposed system of equations is too low. Furthermore, it is unrealistic to assume that $d \Phi / d t$ is independent of $r$, and that the relaxation back to the limit cycle is instantaneous. Finally, due to the rotational symmetry inherent in the model, the PTC shows symmetries (see Section III) which are not observed experimentally (Pavlidis, 1973; Jalife and Moe, 1979; Scott, 1979; Guevara et al., 1981). Possible changes in the behaviour of the system which arise from relaxing one or more of our assumptions to make them more realistic have not yet been studied.

The PTC describes the response of the oscillator to an isolated impulse. The PTC can be used to predict the response to periodic stimulation. In Section IV we show how the PTC can be used to derive a mathematical function called the Poincaré map. Iteration of the Poincaré map allows us to study the dynamics of the model in response to periodic input. Although equivalent procedures have been previously employed (Perkel et al., 1964; Moe et al., 1977; Pinsker, 1977; Glass 
and Mackey, 1979; Scott, 1979; 1980; Levi, 1981), there has not been a thorough analysis of the different phase locking behaviors displayed by a mathematical model displaying both type 0 and type 1 PTC's.

The major results of this paper are given in Fig. 2 which shows the principal phase locking zones as a function of the magnitude $b$ of the perturbation and the time $\tau$ between successive stimuli. Figure 2a shows the zones of $N: M$ phase locking for $N \geqslant M, N \leqslant 3$. Figure $2 \mathrm{~b}$ shows some of these zones as well as all the $4: M$ locking zones over a more limited region of $(\tau, b)$ parameter space. In the unlabelled regions of Fig. 2, there are other phase locked zones, as well as points at which phase locked dynamics does not occur (see Section V).

Fig. 2. Phase locking zones resulting from periodic pulsatile inputs of magnitude $b$ and frequency $\tau^{-1}$. The areas not labelled contain both phase locked and non-phase locked dynamics (see Section V). (a) Phase locking zones of the form $N \geqslant M, N \leqslant 3$. (b) Phase locking zones of the form $N \geqslant M, N \leqslant 4$ over a more limited region of $(\tau, b)$ parameter space. Using the symmetry relations in Section IVB, phase locking patterns for other values of $\tau$ can be generated
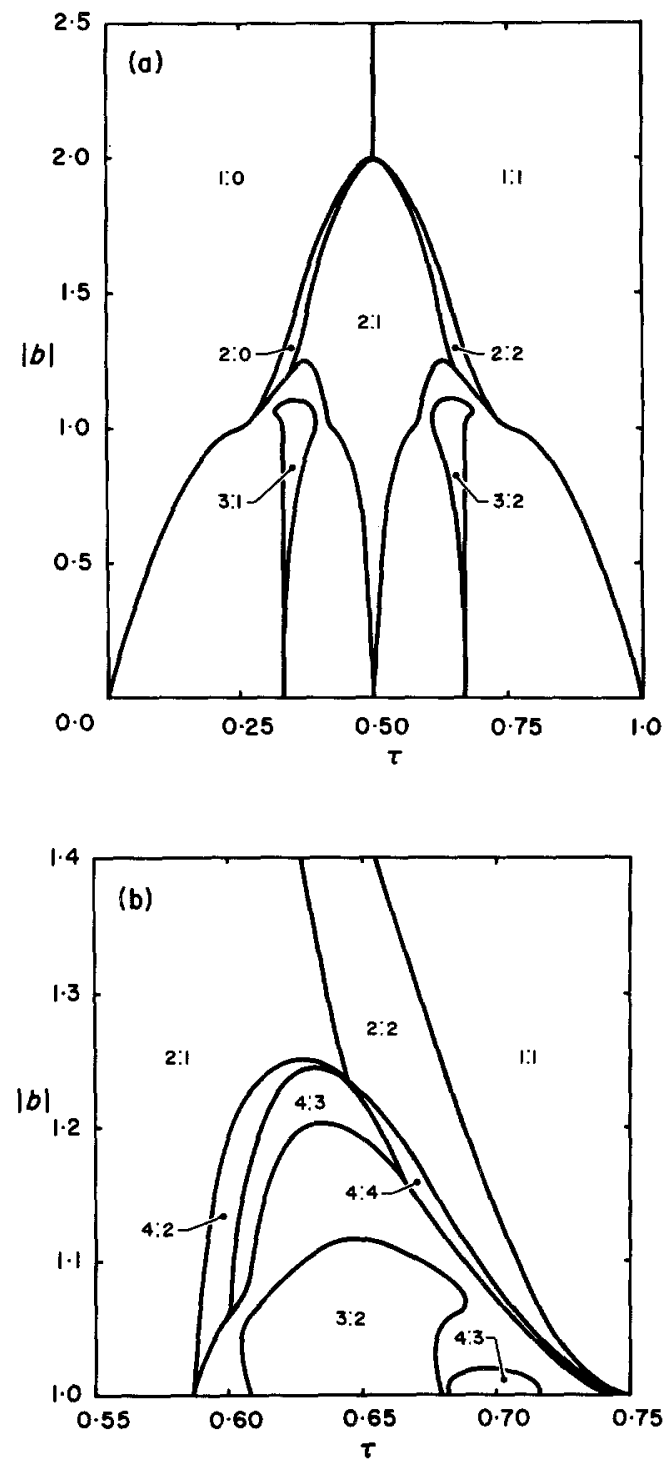
In order to illustrate the different phase locking patterns, we assume that crossing of the positive $x$-axis (i.e. $\phi$ passing through 0 ) corresponds to an observable event (for example, an action potential). In Figs. 3 and 4 we show some of the different coupling patterns between the periodic perturbation and the model oscillator at several different values of frequency and amplitude of the perturbation. The periodic input pulses are represented in Figs. 3 and 4 by heavy dark lines and the times when the model oscillator passes through the phase $\phi=0$ (called the firing times) are represented by lighter, shorter lines. All the patterns shown are phase locked except for those in Fig. 3b and Fig. 4d. The rhythm in Fig. 3b is an example of quasiperiodic dynamics (Glass et al., 1980), and the rhythm in Fig. 4d is an example of an irregular pattern arising out of chaotic dynamics (see Section V).

There is a striking qualitative similarity between the patterns observed in Figs. 3 and 4 and coupling patterns observed between periodic input and driven activity in experimental preparations (Perkel et al., 1964; Reid, 1969; Pinsker, 1977; Jalife and Moe, 1979; Guttman et al., 1980). Many of the patterns in Figs. 3 and 4 resemble clinically observed cardiac arrhythmias such as AV block (see Section VI).
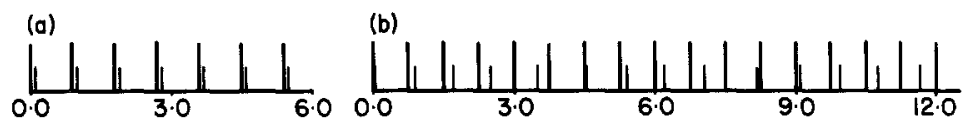

(c)

(d)

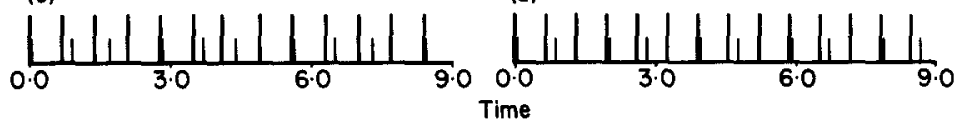

Fig. 3. Schematic representation of the effects of periodic stimulation on the model oscillator for $b=0.95$. The heavy dark bar shows the periodic pulsatile input and the shorter light bar shows the firing times (time when oscillator passes through phase $\phi=0$ ) of the model oscillator. (a) $\tau=0.90,1: 1$ phase locking; (b) $\tau=0.75$, quasiperiodic dynamics; (c) $\tau=0.70,4: 3$ phase locking; (d) $\tau=0.65,3: 2$ phase locking. The patterns of (c) and (d) display Wenckebach periodicity, since the interval between stimulus and succeeding response gets progressively longer until the driven oscillator skips or misses a beat
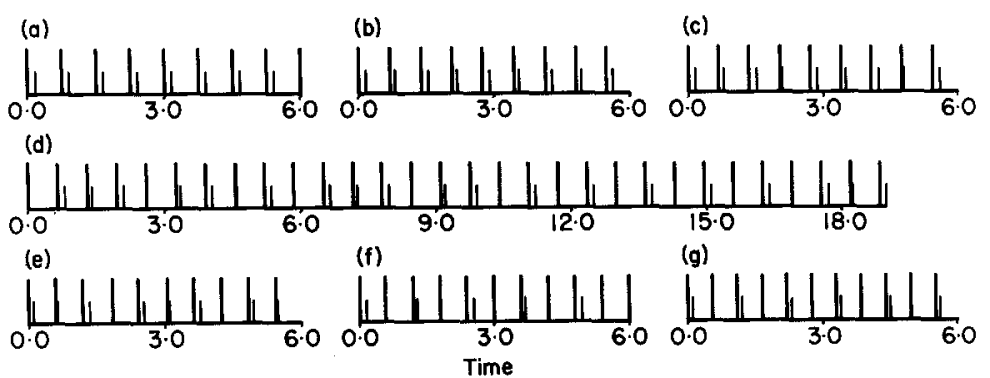

Fig. 4. Same as Fig. 3 but with $b=1$.13. (a) $\tau=0.75,1: 1$ phase locking; (b) $\tau=0.69,2: 2$ phase locking: note the alternation of firing times; (c) $\tau=0.68,4: 4$ phase locking: note that there are four different firing times; (d) $\tau=0.65$, irregular coupling arising from chaotic dynamics: note the narrow range of firing times and the irregularly skipped beats of the driven oscillator; (e) $\tau=0.607,4: 3$ phase locking: note the atypical Wenckebach periodicity; (f) $\tau=0.60,4: 2$ phase locking: note again the alternation of firing times; $(\mathrm{g}) \tau=0.55,2: 1$ phase locking 


\section{The Phase Transition Curve (PTC) and Its Properties}

The PTC gives the new phase $\theta$ as a function of the old phase $\phi$, following a perturbation of amplitude $b$ (Fig. 1). The computations which follow require analytical expressions for the PTC. The PTC is written using the principal values of the inverse tangent and inverse cosine functions, denoted by $\tan ^{-1} x$ and $\cos ^{-1} x$ respectively. For $-\infty<x<\infty,-\pi / 2<\tan ^{-1} x<\pi / 2$ and for $-1<x<1$, $0<\cos ^{-1} x<\pi$. From the construction in Fig. 1 the PTC is readily computed. Define

$$
\begin{aligned}
& \alpha=\frac{1}{2 \pi} \tan ^{-1}\left(\frac{\sin 2 \pi \phi}{\cos 2 \pi \phi+b}\right), \\
& \beta=\frac{1}{2 \pi} \cos ^{-1}(-b) .
\end{aligned}
$$

We find

$$
\begin{array}{ccc}
f(\phi, b)=\alpha, & 0 \leqslant \phi \leqslant \beta, & |b| \leqslant 1, \\
f(\phi, b)=0.5+\alpha, & 0 \leqslant \phi \leqslant 0.5, & b>1, \\
& \beta<\phi<1-\beta, & |b| \leqslant 1, \\
f(\phi, b)=1.0+\alpha, & 0 \leqslant \phi<1, & b<-1, \\
1-\beta \leqslant \phi<1, & |b| \leqslant 1, \\
0.5<\phi<1, & b>1 .
\end{array}
$$

Figure 5 shows examples of type 1 PTCs (which are found for $|b|<1$ ) and type 0 PTCs (which are found for $|b|>1$ ). The PTCs are continuous on the unit circle and have continuous derivatives of all orders on the unit circle. Mathematical models displaying PTCs which are discontinuous on the unit circle have been described (Glass and Mackey, 1979; Keener, 1980; Keener et al., 1981).

The PTCs given by (5) and shown in Fig. 5, display the following symmetries

$$
\begin{aligned}
f(1-\phi, b) & =1-f(\phi, b), & & \\
f(\phi+0.5,-b) & =f(\phi, b)+0.5, & & \phi \leqslant 0.5, \\
f(\phi-0.5,-b) & =f(\phi, b)-0.5, & & \phi \geqslant 0.5 .
\end{aligned}
$$

Fig. 5. The phase transition curve (PTC) of the model oscillator for several values of $b$. For $|b|<1$ the PTC has average slope 1 and is called a type 1 PTC, whereas for $|b|>1$ the PTC has average slope 0 and is called a type 0 PTC

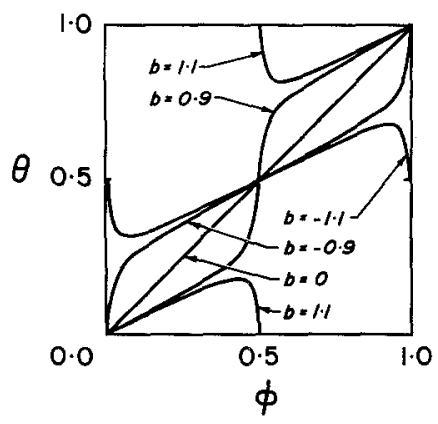


These symmetry relations in the PTC will be used later to derive symmetries in the phase locking zones.

The slope of the PTC is given by

$$
\frac{\partial f}{\partial \phi}=\frac{1+b \cos 2 \pi \phi}{1+b^{2}+2 b \cos 2 \pi \phi} .
$$

For $|b|<1$, the PTC is a monotonically increasing function on the interval $(0,1)$. For $|b|>1$ there is a unique local minimum at $\phi_{\min }$ and a unique local maximum at $\phi_{\max }$.

Define

$$
\begin{aligned}
& \mu=\frac{1}{2 \pi} \cos ^{-1} \frac{1}{|b|}, \\
& \nu=\frac{1}{2 \pi} \tan ^{-1}\left(b^{2}-1\right)^{-1 / 2} .
\end{aligned}
$$

For $b>1$, we compute

$$
\begin{array}{ll}
\phi_{\min }=0.5+\mu, & f\left(\phi_{\min }, b\right)=1-v, \\
\phi_{\max }=0.5-\mu, & f\left(\phi_{\max }, b\right)=v,
\end{array}
$$

and for $b<-1$

$$
\begin{array}{ll}
\phi_{\min }=\mu, & f\left(\phi_{\min }, b\right)=0.5-v, \\
\phi_{\max }=1-\mu, & f\left(\phi_{\max }, b\right)=0.5+v .
\end{array}
$$

These formulae will be used in the later analysis.

\section{The Poincaré Map and Phase Locking}

In this section we use the PTC to construct the Poincaré map in order to investigate the response of the model oscillator to periodic stimulation. The limit $a \rightarrow \infty$ in (1) was taken in order to reduce the Poincaré map to a one dimensional map.

\section{IVA. Fixed Points, Phase Locking, and the Rotation Number}

Call $\phi_{i-1}$ the phase immediately preceeding the $(i-1)$ st stimulus, and $\tau$ the time interval between two consecutive stimuli of the periodic input. Then immediately preceeding the $i$ th stimulus, the phase of the oscillator will change by an amount $\Delta \phi_{i}$, where

$$
\Delta \phi_{i}=f\left(\phi_{i-1}, b\right)+\tau-\phi_{i-1}
$$

and $f\left(\phi_{i-1}, b\right)$ is defined by (5). Consequently, the phase immediately preceeding the $i$ th stimulus is

$$
\phi_{i}=f\left(\phi_{i-1}, b\right)+\tau \quad(\bmod 1) .
$$

The map

$$
T: \phi_{i-1} \rightarrow \phi_{i}
$$


Fig. 6. The Poincaré map for $b=-1.30$ and three values of $\tau$

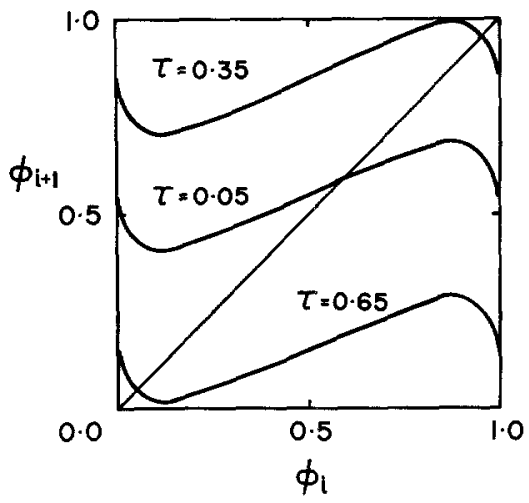

defined by (11) is called the Poincare or phase advance map. Figure 6 shows the Poincaré map for $b=-1.30$ and for three values of $\tau$. This figure illustrates that vertical translation of the PTC by an amount $\tau$ gives the Poincare map.

Iteration of the Poincaré map can be used to compute the evolution of the phase in response to periodic input (Arnold, 1973; Glass and Mackey, 1979; Guckenheimer, 1980; Levi, 1981)

$$
\begin{aligned}
\phi_{i+1} & =T\left(\phi_{i}\right), \\
\phi_{i+m} & =T\left(\phi_{i+m-1}\right)=T^{m}\left(\phi_{i}\right) .
\end{aligned}
$$

A phase $\phi^{*}$ is called a fixed point of period $N$ if

$$
\begin{aligned}
T^{N}\left(\phi^{*}\right) & =\phi^{*}, \\
T^{i}\left(\phi^{*}\right) & \neq \phi^{*}, \quad 1 \leqslant i<N .
\end{aligned}
$$

A fixed point $\phi^{*}$ of period $N$ is stable if

$$
\left|\frac{\partial T^{N}\left(\phi_{i}\right)}{\partial \phi_{i}}\right|_{\phi_{i}=\phi^{*}}<1 .
$$

A concept which has been useful in the study of phase locking is the rotation number, $\rho$, defined as the average advance in phase of the driven oscillator for each cycle of the periodic input. The rotation number is defined

$$
\rho=\lim _{J \rightarrow \infty} \frac{1}{J} \sum_{i=1}^{J} \Delta \phi_{i},
$$

where $\Delta \phi_{i}$ is given in (10).

Stable fixed points on the Poincare map are associated with phase locked dynamics. Assume that for some value of $(\tau, b)$, there are $N$ stable fixed points of period $N, \phi_{0}, \phi_{1}, \ldots, \phi_{N}=\phi_{0}$ where $\phi_{i+1}=T\left(\phi_{i}\right)$ as before. Define

$$
M=\sum_{i=1}^{N} \Delta \phi_{i} .
$$

Since $\phi_{N}=\phi_{0}, M$ must be an integer and the sequence is associated with $N: M$ phase locking with rotation number $\rho=M / N$. Consequently, for phase locked 
patterns, the rotation number is rational. Note that $\rho$ does not specify a unique phase locking pattern. For example, both $1: 1$ and $2: 2$ locking have $\rho=1$ and both $2: 1$ and $4: 2$ phase locking have $\rho=\frac{1}{2}$. Also, for fixed values of $b$ and $\tau, \rho$ may not be unique and may depend on the initial phase.

\section{IVB. Symmetries of the Phase Locking Zones}

As a consequence of the symmetries of the PTC detailed in (6), there are symmetries in the phase locking zones. These symmetries are summarized by the following propositions, the first of which is proven in Appendix A.

Proposition 1. Assume that there is a stable period $N$ cycle with fixed points $\phi_{0}, \phi_{1}, \ldots, \phi_{N-1}$ for $b=\gamma, \tau=0.5-\delta(0<\delta<0.5)$ associated with an $N:$ M phase locking pattern. Then, for $\tau=0.5+\delta$, there will be a stable cycle of period $N$ associated with an $N: N-M$ phase locking ratio. The $N$ fixed points $\psi_{0}, \psi_{1}, \ldots, \psi_{N-1}$ of this cycle are given by

$$
\psi_{i}=1-\phi_{i}
$$

Proposition 2. Suppose that for $b=\gamma, \tau=\delta(0<\delta<1)$ there is a stable cycle of period $N$ with fixed points $\phi_{0}, \phi_{1}, \ldots, \phi_{N-1}$ associated with an $N: M$ phase locking pattern. Then, for $b=-\gamma, \tau=\delta$, there will be a stable cycle of period $N$ associated with an $N: M$ phase locking pattern with $N$ fixed points $\psi_{0}, \psi_{1}, \ldots, \psi_{N-1}$ where

$$
\begin{array}{lr}
\psi_{i}=\phi_{i}+0.5, & 0<\phi_{i}<0.5, \\
\psi_{i}=\phi_{i}-0.5, & 0.5 \leqslant \phi_{i}<1.0 .
\end{array}
$$

Proposition 3. Suppose there is a stable cycle of period $N$ with fixed points $\phi_{0}, \phi_{1}, \ldots, \phi_{N-1}$ for $b=\gamma, \tau=\delta(0<\delta<1)$ associated with an $N$ : M phase locking pattern. Then there will be a stable cycle of period $N$ with fixed points $\phi_{0}, \phi_{1}, \ldots, \phi_{N-1}$ for $b=\gamma, \tau=\delta+K$ (where $K$ is any positive integer) associated with an $N: M+K N$ phase locking ratio.

\section{IVC. The Poincare Map as an Interval Map with a Single Maximum}

Over certain regions of the $(\tau, b)$ parameter space, the Poincaré map reduces to a map defined on an interval in which there is a single maximum of the map. Interval maps with a single maximum have been the focus of considerable analysis recently (Li and Yorke, 1975; May, 1976; Guckenheimer, 1977; Štefan, 1977). The boundaries of the phase locking zones shown in Fig. 2 can be partially understood by considering the bifurcations of interval maps.

The reduction of the Poincare map to an interval map with a single maximum can be illustrated by considering an example. Figure 6 shows the Poincare map for $\tau=0.35, b=-1.30$. For this situation, $T:[0,1] \rightarrow[0.7103,0.9897]$ and in this interval $T$ has a single maximum (Fig. 7). In general, for $b<-1$ and $0 \leqslant \tau \leqslant 0.5$ the first iterate of the Poincare map will be an invariant interval map with a single maximum provided the following inequalities hold: 

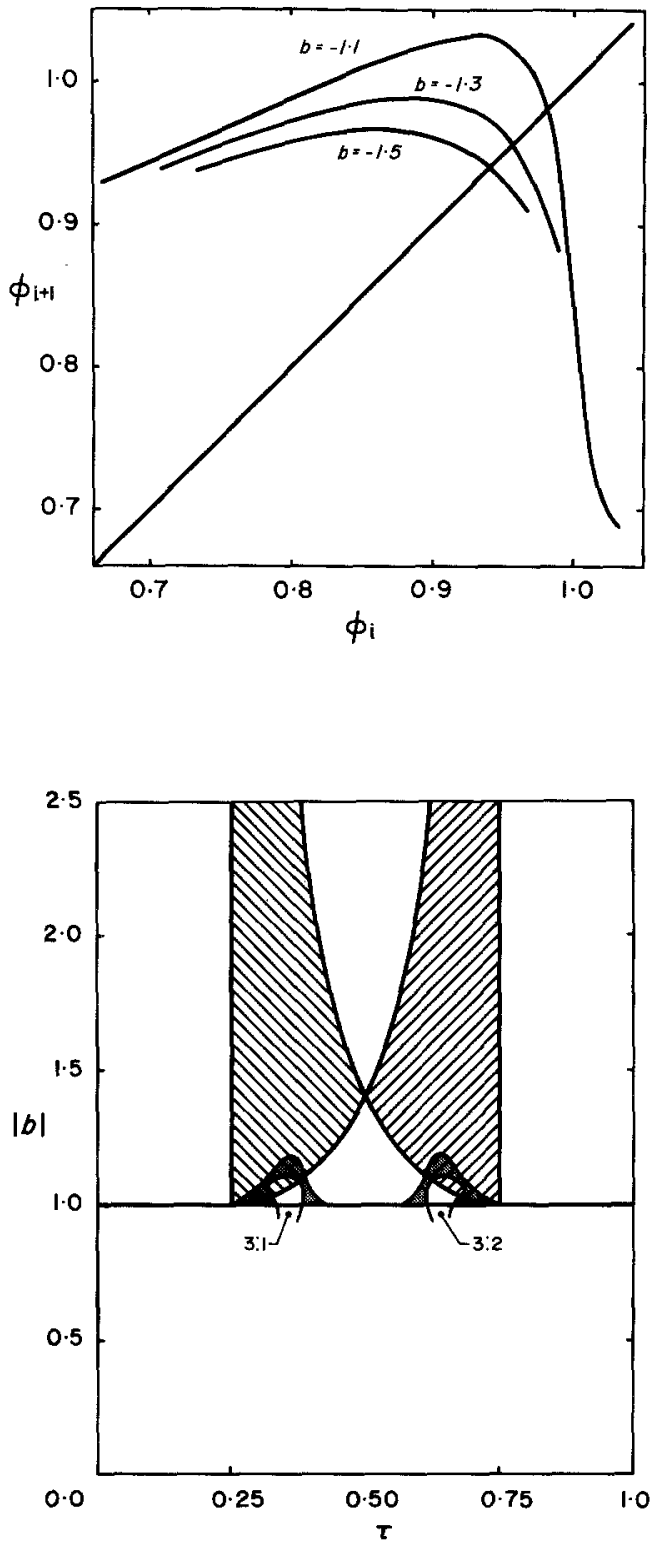

Fig. 7. The Poincaré map for $\tau=0.35$ and three values of $b$. For each value of $b$ all initial phases between 0 and 1 will map into the invariant region shown following one iteration of the Poincare map. For ease of presentation, $\phi_{i+1}$ is not given modulo 1

Fig. 8. Representation of the three regions in which qualitatively different dynamics occur. In the diagonally striped area the Poincare map reduces to an interval map with a single maximum (Section IVC). For $|b|<1$ (region I) the Poincare map is monotonic, continuous and differentiable on the unit circle. In this region there will either be quasiperiodic or phase locked dynamics. For $|b|>1$ there are two qualitatively different regions with respect to the types of transitions between neighboring phase locking zones. Region III is comprised of the small stippled areas as well as the $3: 1$ and $3: 2$ zones, whereas region II is the remainder of the area for $|b|>1$. In region II are found period doubling bifurcations leading to phase locking ratios of the form $2^{i}: M$. The complex dynamics of region III are discussed in the text (Section VC) 


$$
\begin{aligned}
& \tau+f\left(\phi_{\max }, b\right)>\phi_{\max } \\
& \tau+f\left(\phi_{\max }, b\right)<\phi_{\min } \\
& \tau+f\left(\phi_{\min }, b\right)<\phi_{\max }
\end{aligned}
$$

Applying some simple trigonometry, the inequalities can be rewritten using (8) and (9)

$$
\begin{aligned}
\tau & >0.25, \\
\tau & <\frac{1}{2}-\frac{1}{2 \pi} \sin ^{-1}\left|\frac{b^{2}-2}{b^{2}}\right|,
\end{aligned}
$$

where $\sin ^{-1} x$ denotes the principal value of the inverse sine function $\left(-\pi / 2<\sin ^{-1} x<+\pi / 2\right.$ for $\left.-1<x<1\right)$. From the symmetries in (6), the Poincaré map in other regions of $(\tau, b)$ parameter space can also be reduced to an interval map with a single maximum. In Fig. 8 the regions of $(\tau, b)$ parameter space in which the Poincare map reduces to an interval map with a single maximum are shown by diagonal stripes.

In Fig. 7 we show the Poincaré map for $\tau=0.35, b=-1.1,-1.3,-1.5$ over the region in which the map is an interval map with a single maximum. Note that as $|b|$ approaches 1 , the map becomes progressively steeper in the neighborhood of the fixed point $\phi_{i+1}=\phi_{i}$. Note from Fig. 6 that changes in $\tau$ while keeping $b$ constant also can lead to changes in the slope of the map at the fixed point. As we will show below, a progressive steepening of the slope at the fixed point is associated with a sequence of period doubling bifurcations that lead to chaotic dynamics.

\section{Phase Locking Zones in Different Regions of $(\tau, b)$ Parameter Space}

Our major goal has been to provide qualitative and quantitative analysis of the different types of dynamics that arise from pulsatile periodic inputs to a simple two dimensional limit cycle oscillator (Fig. 1). The analysis has involved numerical study of the effects of iteration of the Poincare map at different values of $(\tau, b)$ as well as analytic derivation of bifurcation boundaries when this has been feasible. The analytic computations are discussed in Appendix B. These computations were cross-checked with numerical computation. For a given value of $b$, the values of $\tau$ at the boundaries of the phase locking zones are accurate to \pm 0.01 in Fig. $2 \mathrm{a}$ and to \pm 0.003 in Fig. $2 b$.

The analysis shows that there are three different regions of $(\tau, b)$ parameter space that can be identified with respect to the types of transitions between neighboring phase locking zones. The three regions are shown in Fig. 8. The types of bifurcations that occur in each region will now be briefly discussed.

\section{$V A$. Region $I:|b|<1$}

In this region the Poincaré map is a continuous, monotonic and differentiable map of the unit circle onto itself. The qualitative properties of the dynamics can be completely described by the rotation number $\rho$. If $\rho$ is rational then there is 
stable phase locking (Figs. 3a, 3c, 3d) whereas if $\rho$ is irrational, quasiperiodic dynamics result (Fig. 3b). In quasiperiodic dynamics, there is a continual shift of the firing time with respect to the stimulus.

In region $\mathrm{I}$, the rotation number is a continuous function of $\tau$ and $b$ which is piecewise constant over the set of rationals. The measure of the set on which the rotation number is irrational is positive (Herman, 1977). Thus, the dynamics found in region I are topologically equivalent to the dynamics found in other models of phase locking in the limit of "small" perturbations (Arnold, 1965; Knight, 1972; Glass and Mackey, 1979; Keener et al., 1981). These references should be consulted for a further discussion of the dynamics in region I.

\section{VB. Region II: Period Doubling Bifurcations with $|b|>1$}

In region II, there are orbits of period $2^{i}$ ( $i$ a non-negative integer). There are two different types of boundaries between adjacent phase locking zones: boundaries due to period doubling bifurcations, and boundaries due to changes in the rotation number. The analytic computation of boundaries in region II is discussed in detail in Appendix B.

On a period doubling boundary, there is a period doubling bifurcation of the associated Poincaré map without any change in rotation number. For example, such period doubling bifurcations are responsible for the borders between the 1:0 and $2: 0$ zones and the $1: 1$ and $2: 2$ zones of Fig. $2 a$, and between the $2: 1$ and $4: 2$ zones and the $2: 2$ and $4: 4$ zones of Fig. $2 b$. If $\phi^{*}$ is a fixed point of the Poincare map $T^{N}$, then a period doubling (or "pitchfork") bifurcation to an orbit of period $2 N$ will arise when

$$
\left.\frac{\partial T^{N}}{\partial \phi}\right|_{\phi=\phi^{*}}=-1 .
$$

In Fig. 9 we show the stable fixed points for $\tau=0.35$ as a function of $b$. For $-1.8<b<-1.0$ there is the familiar cascading sequence of fixed points culminating in the emergence of an orbit of period 3, analogous to the sequences found in studies of the iteration of one parameter families of interval maps with a single maximum (May, 1976; Guckenheimer, 1977).

Fig. 9. The stable fixed points as a function of $b$ for $\tau=0.35$. Only the first two period doubling bifurcations and the period 3 orbit are shown. Lying in the blank space between the period 4 orbit and the period 3 orbit are orbits of all other periods (May, 1976; Guckenheimer, 1977). For ease of presentation, $\phi$ is not given modulo 1

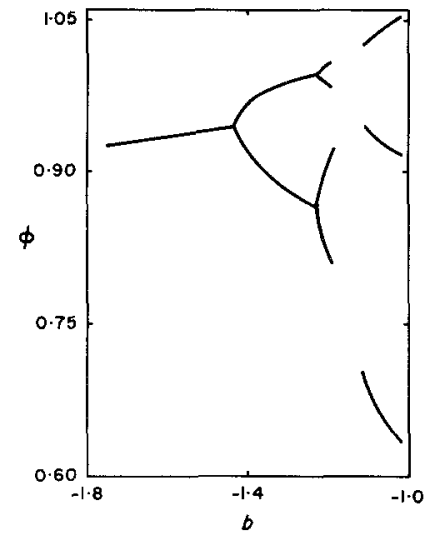




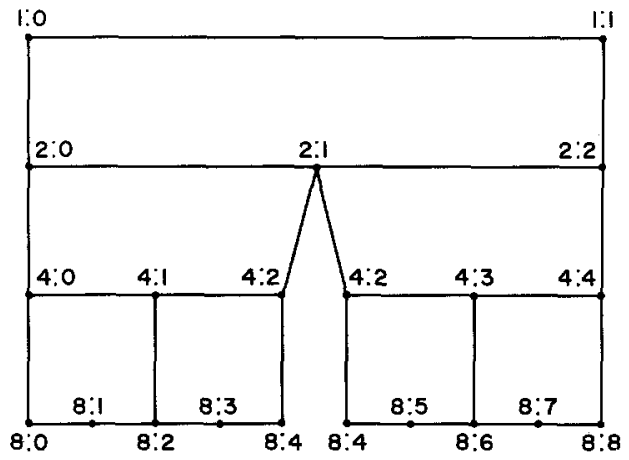

Fig. 10. Hypothesized arrangement of the phase locking zones in region II of Fig. 8. The vertices represent the phase locking zones and the edges represent the boundaries between neighboring zones. Along each horizontal line the rotation number increases. Vertical edges represent phase locking boundaries corresponding to period doubling bifurcations of the Poincaré map

Delicately interlaced with these period doubling boundaries are boundaries across which the rotation number changes, but across which the number of fixed points on the Poincare map remains constant. For example, such boundaries occur between the $1: 0$ and $1: 1$ zones (Fig. 2a), the $2: 1$ and $2: 2$ zones (Fig. 2a, 2b), the $4: 2$ and $4: 3$ zones (Fig. 2b), and the $4: 3$ and $4: 4$ zones (Fig. 2b). If $\phi^{*}$ is a fixed point of the map $T^{N}$ for $b<-1$, then such a boundary occurs when

$$
\phi^{*}=0 .
$$

Note that in Fig. 9, $\phi^{*}=0(=1, \bmod 1)$ for $b \simeq-1.220, \tau=0.35$. This point lies on the boundary of the $4: 0$ and $4: 1$ phase locking regions.

The two distinct types of boundaries intersect at singular points at which (22) and (23) are simultaneously satisfied. The singular points represent points on the common boundaries of five different phase locking zones. The point $|b|=2$, $\tau=0.5$, which is on the boundaries of the $1: 0,1: 1,2: 2,2: 1$, and $2: 0$ phase locking regions (Fig. 2a) represents such a singular point. Applying (22) and (23) enables us to compute the singular point at $|b|=2, \tau=0.5$ as well as a pair of singular points located at $|b|=5^{1 / 2}-1, \tau \simeq 0.5 \pm 0.14$ (Fig. $2 \mathrm{~b}$, Appendix B). In addition to these singular points, numerical studies show that there are four additional singular points at which period 8 orbits arise located at the values $|b| \simeq 1.17, \tau \simeq 0.5 \pm 0.16$ and $|b| \simeq 1.06, \tau \simeq 0.5 \pm 0.10$.

On the basis of these results, we propose that the topology of the different phase locking zones and singular points in region II can be depicted using the graphical representation shown in Fig. 10. This graph is the geometric dual of the phase locking zones in the period doubling region of Fig. 2. Thus vertices of the graph represent the phase locking zones, while the edges of the graph represent the boundaries between phase locking zones. Note that each row of the graph is related to the rows above it by period doubling bifurcations. Within each row the rotation number increases as one progresses from left to right. Numerical studies have not been performed to check the validity of the extension of the scheme in Fig. 10 to orbits of period $2^{i}, i \geqslant 4$.

\section{VC. Region III: Stippled Areas Plus 3:1 and 3:2 Zones in Fig. 8}

In region III, there is an overlap between the regions where the Poincare map reduces to an interval map with a single maximum (the diagonally striped regions of 
Fig. 8) and the regions where stable period 3 orbits exist (corresponding to $3: 1$ and $3: 2$ phase locking). A consequence of this fact is that "chaos" exists at values of $(\tau, b)$ where such a period 3 orbit exists, in that there are initial phases starting from which iteration of the Poincaré map will produce orbits of arbitrarily large period as well as aperiodic orbits (Šarkovskiĩ, 1964; Stefan, 1975; Li and Yorke, 1975). However, these orbits are unstable, and the sole attracting orbit is the one of period 3. Thus, in a physical or biological system, one would not observe any of the infinity of unstable orbits, but only the attracting one of period 3 .

We expect that the area of $(\tau, b)$ space lying between the period doubling zone of region II and the period 3 orbits (i.e. the stippled area of Fig. 8) will contain $N: M$ phase locking zones with arbitrary $N$, as well as points where aperiodic patterns exist (Metropolis et al., 1973; May, 1976; Guckenheimer, 1977; Hoppensteadt, $1981)$. For most values of $(\tau, b)$ in this region, we find complicated dynamics with an irregular appearance such as that shown in Fig. $4 \mathrm{~d}(b=1.13, \tau=0.65)$. This pattern shows no sign of periodicity after 1000 iterations. Note that the irregularity is manifest by a seemingly random dropping or skipping of beats of the driven oscillator, even though the intervals between stimuli and firing times are restricted to a comparatively narrow range.

The term "chaotic dynamics" is appropriate to describe the extremely complex behaviour displayed by the model in the stippled area of Fig. 8. As discussed above, the pattern generated at a particular value of $(\tau, b)$ in this area is in general very complicated and may even be aperiodic. In addition, since quite small changes in $\tau$ or $b$ away from that particular value of $(\tau, b)$ will cause a change in the pattern observed, the effect of fluctuations or "noise" inherent to any experimental system will be to destroy patterns in zones with high order phase locking ratios (Crutchfield and Huberman, 1980; Glass et al., 1980; Guttman et al., 1980). Thus, higher order zones that cover small enough areas of $(\tau, b)$ parameter space will not be observed in the laboratory. Instead, they will be replaced by zones that are not phase locked and which display irregular dynamics.

Orbits in region III (such as the period 3 orbit) can arise from tangent bifurcations (see Appendix B). The detailed topology of the phase locking zones in this region is not well understood.

\section{Discussion}

We are interested in mechanisms for the genesis of cardiac arrhythmias, and have been conducting experiments in which spontaneously beating aggregates of cardiac cells are periodically stimulated with pulses of current (Guevara et al., 1981). Our development of the simple mathematical model in this paper was motivated by a desire to obtain insight into the dynamics of an oscillator in response to periodic stimulation. We have found that the experimental preparation behaves in a manner that is similar to the behavior of the model. In particular, the experimentally determined Poincaré maps can sometimes be reduced to interval maps containing a single maximum. When iterated, these maps show evidence of period doubling bifurcations and chaotic dynamics. Furthermore, in response to periodic stimulation, patterns due to period doubling bifurcations (such as $2: 2$ and $4: 4$ phase 
locking) are experimentally found, as well as irregular patterns which may be due to underlying "chaotic dynamics".

The experimental observation of these phenomena in cardiac tissue, coupled with the predictions of a rather general mathematical model, leads us to believe that the results of this study have direct implication for the analysis of both normal and pathological cardiac rhythms. Under normal physiological conditions, the primary pacemaking site in the heart is located in the sinoatrial (SA) node. The cardiac impulse originates in the SA node, spreads to the atrial musculature, proceeds to the atrioventricular (AV) node, and then passes through the bundle of His, the bundle branches, and the Purkinje network to the ventricular muscle (Mandel, 1980). Thus, firing of the SA node leads to an almost immediate contraction of the atria, followed after a small delay by contraction of the ventricles.

There are at least two ways in which to interpret the delay between activation of the atria and the ventricles. The traditional and most widely held view is that the delay is due to slow conduction of the cardiac impulse when passing through the AV node (Tsien and Siegelbaum, 1978).

One alternate view is based on the hypothesis that the specialized electrical conduction system of the mammalian heart contains two or more autonomous oscillators (van der Pol and van der Mark, 1928). Normally, one oscillator (situated in the SA node) is the driving oscillator that entrains another (situated in the region of the AV node) in a 1:1 fashion. Recent work has provided simultaneous. anatomical and electrophysiological evidence that the site of the driven (subsidiary or latent) oscillator is at the border of the AV node and the bundle of His (James et al., 1979). Since the driven oscillator has an intrinsic frequency that is lower than that of the SA node (Nadeau and James, 1966; Urthaler et al., 1973; Urthaler et al., 1974), there will be a phase shift between the two oscillators when synchronization in a 1:1 pattern occurs (Fig. 3a, 4a). This phase shift would appear as a delay between the contraction of the atria and the contraction of the ventricles. Indeed, if the intrinsic frequency of the SA node is decreased sufficiently by pharmacological intervention, the phase shift reverses its sign, and contraction of the ventricles precedes contraction of the atria (Nadeau and James, 1966; Roberge et al., 1968).

Modeling the heart as a system of two or more coupled nonlinear oscillators was pioneered by van der Pol and van der Mark (1928) using an electrical circuit. In the intervening 50 years, several other investigators have extended their work using electrical and electronic analogues and computer simulations (Grant, 1956; Roberge et al., 1968; Sideris and Moulopoulos, 1977; Katholi et al., 1977) as well as physiological experimentation (Nadeau and James, 1966; Roberge et al., 1968; Roberge and Nadeau, 1969; Urthaler et al., 1973; Urthaler et al., 1974). The modeling work shows that the disturbances of atrioventricular conduction (AV blocks) seen in the electrocardiogram can be simulated by changing either of the intrinsic frequencies of the two oscillators or by altering the degree of interoscillator coupling. The physiological work also demonstrates that pharmacological manipulation of the intrinsic frequencies of the oscillators or of the level of block at the AV node can produce dysrhythmias when 1:1 synchronization is lost. Prior modelling largely relied on electrical analogues. However, there has not been a theoretical investigation and an analysis of the topology of the phase locking zones such as that presented here. 
In electrocardiography, electrical events associated with the contraction of the atria ( $P$ wave) and ventricle (QRS complex) can be observed by recording electrical potentials on the surface of the body. The coupling patterns between the stimulus and oscillator observed in Figs. 3 and 4 are similar to clinically observed patterns of AV block. In particular, we make the following identifications:

i) Normal sinus rhythm or first degree AV block - Fig. 3a, Fig. 4a.

ii) $1: 1 \mathrm{AV}$ conduction with alternating PR intervals - Fig. $4 \mathrm{~b}(2: 2 \mathrm{block})$.

iii) Second degree AV block with Wenckebach periodicity-Fig. 3c $(4: 3$ block), Fig. 3d (3:2 block).

iv) Second degree AV block with atypical Wenckebach periodicity - Fig. 4e ( $4: 3$ block).

v) Second degree AV block with variable conduction or third degree AV block with accrochage - Fig. 4 d.

vi) High grade second degree AV block - Fig. 4g (2:1 block).

vii) Second degree AV block (2:1 block with alternating PR intervals) - Fig. $4 \mathrm{f}$ ( $4: 2$ block).

viii) Third degree or complete AV block - Fig. $3 b$.

The current analysis predicts the existence on purely theoretical grounds of such patterns as 2:2 and 4:2 phase locking, whereas prior theoretical work did not identify these patterns. A striking description of a $2: 2$ pattern is in an early canine study by Lewis and Mathison (1910) of the results of asphyxia on the heart beat: "At or about the time when the heart appears to waver between a condition of $2: 1$ heart block and regular sequential contraction accompanied by prolongation of the P-R interval, it not infrequently happens that passing into the latter state it exhibits a regular alternation of the P-R intervals" (see Fig. 5 of Lewis and Mathison (1910) and compare with Fig. $4 \mathrm{~b}$ of this paper). A recent review (Watanabe and Dreifus, 1980) has emphasized that the mechanisms for generating patterns with alternating PR intervals ( $2: 2$ rhythms) are not well understood and are controversial. Note that for $5^{1 / 2}-1<b<2$, as the frequency of the stimulation is increased one passes in turn from $1: 1$ to $2: 2$ to $2: 1$ phase locking (Fig. 2). Thus, we hypothesize that the pattern with alternating PR intervals experimentally observed corresponds to a $2: 2$ phase locking pattern and arises as a consequence of a period doubling bifurcation. The $4: 2$ patterns clinically observed (Segers, 1951) may be due to a second period doubling bifurcation (Fig. 4f).

Another feature of our analysis is that chaotic dynamics can arise as a consequence of periodic inputs to a limit cycle oscillator. A review of clinically observed dysrhythmias reveals that there are many dysrhythmias which have an extremely irregular appearance. To cite a few examples of irregular AV block:

i) "Advanced second degree block with fluctuation in the depth of penetration into the AV node" - Fig. 16-17 in Watanabe and Dreifus (1980).

ii) "Intermittent $2: 1 \mathrm{~A}-\mathrm{V}$ block and an area of $3: 2$ Mobitz type II A-V block" - Fig. 291 in Chung (1971).

iii) "Wenckebach A-V block of varying degree (6:5, 5:4, 4:3, 3:2)" - Fig. 295 in Chung (1971).

iv) "Atrial flutter with varying second degree AV block and phasic aberrant ventricular conduction"-Case 8, p. 349 in Schamroth (1971). 
v) "Partial AV heart block" - Fig. 1-16E in Bellet (1971).

These examples serve to illustrate the fact that clinically observed cardiac dysrhythmias are frequently quite irregular. On the basis of our analysis, we propose that one source of irregularity in electrocardiographic patterns of $\mathrm{AV}$ block may well be chaotic dynamics resulting from interaction between different autonomous pacemaker sites in the heart (Fig. 4d). Testing of this hypothesis requires collection and analysis of long electrocardiographic records, as well as a better understanding of the different ways in which chaotic dynamics may become manifest in mathematical models.

Our identification of cardiac dysrhythmia with desynchronization of autonomous oscillators is highly speculative. The reader should be aware that these ideas run counter to conventional clinical teaching, and also that alternative theoretical models based on fatigue and relative refractoriness (Landahl and Griffeath, 1971; Keener, 1981a, 1981b) display some qualitative features similar to the properties described here. However, the detailed topological structure of the phase locking presented in these other models is completely different from that of our model (Keener, 1980, 1981a, 1981b). For example, other models do not include phase locking zones of the form $2 N: 2 M$ and the "chaos" observed by Keener does not arise from a sequence of period doubling bifurcations. Finally, although the theory is general, the above discussion has centered on dysrhythmias arising in the AV conduction system. For instance, interactions between nodal pacemakers and ectopic spontaneously active foci would lead to similar dynamics and dysrhythmias (Moe et al., 1977; Jalife and Moe, 1979).

In the mathematical literature, although it has long been known that aperiodic solutions can be found in response to periodic inputs to two dimensional oscillators (Cartwright and Littlewood, 1945; Levinson, 1949) the topology of the different phase locking zones under changes in the stimulation parameters is not completely understood (Guckenheimer, 1980; Levi, 1981). The novel contribution of our work is to show that over certain ranges of parameter space, the phase locking problem reduces to a problem in the analysis of interval maps. We show that transitions between phase locking zones are due to period doubling bifurcations, tangent bifurcations, or simply changes in rotation number. We have also proposed a scheme for the topology of the phase locking zones (Fig. 10) in the period doubling region. Period doubling bifurcations and chaotic dynamics have been observed in previous work on sinusoidally forced nonlinear oscillators (Tomita and Kai, 1978; Huberman and Crutchfield, 1979). In addition, "chaotic dynamics" have been observed from two mutually coupled tunnel diode oscillators but no evidence of period doubling bifurcations was found (Gollub et al., 1980). However, a study of mutual entrainment of two electronic models of cardiac pacemaker cells apparently did not show chaotic dynamics (Ypey et al., 1980).

One of the main ways for studying biological oscillators is to subject the oscillators to periodic pulsatile inputs. This paper has described the qualitative properties of phase locking for a simple mathematical model of a two dimensional oscillator. On the basis of this theoretical work we predict that period doubling bifurcations leading to chaotic dynamics as a consequence of periodic stimulation of spontaneously oscillating biological systems will be widespread. 
Acknowledgments. This research has been supported by grants from the Natural Sciences and Engineering Research Council of Canada and the Canadian Heart Foundation. We thank M. Mackey and C. Graves for helpful discussions, S. James for typing the manuscript, and B. Gavin for drafting the figures.

\section{Appendix A}

In this appendix we prove Proposition 1 of Section IVB. The proofs of the other two propositions follow in a similar fashion.

Proof of Proposition 1. From the statement of the proposition and the definition of the Poincare map we know that

$$
\begin{aligned}
\phi_{i} & =f\left(\phi_{i-1}, \gamma\right)+0.5-\delta \\
\Delta \phi_{i} & =f\left(\phi_{i-1}, \gamma\right)+0.5-\delta-\phi_{i-1} \\
M & =\sum_{i=1}^{N} \Delta \phi_{i} .
\end{aligned}
$$

Starting from phase $\psi_{i-1}=1-\phi_{i-1}$ and assuming $\tau=0.5+\delta$ we compute the new phase, $\eta$

$$
\eta=f\left(1-\phi_{i-1}, \gamma\right)+0.5+\delta \quad(\bmod 1) \text {. }
$$

Applying the symmetry in Eq. (6a) and substituting from Eq. (A1), we find

$$
\eta=1-\phi_{i} \text {. }
$$

Thus, the sequence $\psi_{0}, \psi_{1}, \ldots, \psi_{N-1}$ will form a cycle for $\tau=0.5+\delta$ where $\psi_{i}=1-\phi_{i}$. The phase locking pattern is computed by summing the $\Delta \psi_{i}$, where

$$
\Delta \psi_{i}=f\left(\psi_{i-1}, \gamma\right)+0.5+\delta-\psi_{i-1}
$$

Once again using the symmetry in (6a) and substituting from (A2), we find

$$
\Delta \psi_{i}=1-\Delta \phi_{i}
$$

Summing and applying (A3), we compute

$$
\sum_{i=1}^{N} \Delta \psi_{i}=N-M
$$

Therefore, the sequence $\psi_{0}, \psi_{1}, \ldots, \psi_{N-1}$ is associated with an $N: N-M$ phase locking pattern.

\section{Appendix B}

In this appendix we give a brief summary of the analytic computation of the boundaries of the phase locking zones and singular points in $(\tau, b)$ parameter space. In this analysis we utilize results from local bifurcation theory in conjunction with a consideration of the rotation number.

Assume that $\phi^{*}$ is a periodic point of period $n$ of the Poincare map (13). Then, change in the stability of the periodic point (bifurcation) will occur if

$$
\left.\frac{\partial T^{n}}{\partial \phi}\right|_{\phi=\phi^{*}}= \pm 1 .
$$

If the derivative in (B1) is +1 the bifurcation is called a saddle-node or tangent bifurcation and if the derivative is -1 then the bifurcation is called a pitchfork of flip bifurcation (May, 1976; Guckenheimer, 1977). In addition to satisfying (B1) there are additional non-degeneracy conditions (Guckenheimer, 1977) which we do not consider in the computations which follow. At a pitchfork bifurcation the number of stable fixed points is doubled but the rotation number is in general the same. At a tangent bifurcation the number of stable fixed points changes and the rotation number may or may not change. 
In addition to changes in rotation number associated with tangent bifurcations, the rotation number of the stable phase locked orbit may change with parametric changes in $b$ and $\tau$, even though the number and stability of the fixed points remain unchanged. Such changes in rotation number only occur for $|b|>1$. To show how these changes arise consider the case in which $b>1$, and assume that along the locus of points

$$
\tau=g(b)
$$

$\phi^{*}=0.5$ is a stable fixed point of period $N$. Assume that in the neighborhood of the curve in (B2) there exists a stable fixed point $\phi^{*}$ of the map $T^{N}$

$$
\phi^{*}=0.5-\varepsilon(b, \tau),
$$

where $\varepsilon$ is a continuous differentiable function

$$
\varepsilon(b, g(b))=0
$$

and $\varepsilon$ is positive on one side of the line in (B2) and negative on the other side. Assume that in the region in which $\varepsilon$ is negative there is $N: M$ phase locking. Then from a direct application of (17) there will be $N: M+1$ phase locking on the other side. Consequently the locus of points in (B2) will constitute the boundary between the two phase locking regions. In a similar fashion, and recalling the symmetries in Section IV, a locus of points along which $\phi^{*}=0$ constitutes a boundary of a phase locking region for $b<-1$.

In the remainder of this Appendix we give a brief summary of the computation of the bifurcation boundaries and singular points in $(\tau, b)$ parameter space. The results are appropriate for $b<0$ and $0 \leqslant \tau \leqslant 0.5$. Using the symmetries in Section IV appropriate formulae for symmetrically placed boundaries can be computed. Since the computations entail simple algebra and trigonometry, only the main results of the computations are given. The formulae presented have been checked against results from numerical iteration of the Poincaré map.

The 1:0 phase locking boundary coincides with a tangent bifurcation for $|b|<1$, a pitchfork bifurcation for $1<|b|<2$ and a change in rotation number for $|b|>2$.

Consider first the locus of the tangent bifurcation. Setting $\partial T / \partial \phi=+1$ and using (7) we find

$$
\phi=1-\frac{1}{2 \pi} \cos ^{-1}(-b) .
$$

Note that the only real solution of (B5) occurs for $|b|<1$, and thus the boundary of the 1:0 phase locking coincides with a tangent bifurcation only for $|b|<1$. Application of (5) and (11) gives the boundary of the $1: 0$ phase locking for $-1<b<0$

$$
\tau=\frac{1}{2 \pi} \cos ^{-1} b-0.25 \text {. }
$$

A pitchfork bifurcation is computed by setting $\partial T / \partial \phi=-1$. Using (7), a pitchfork bifurcation occurs for

$$
\phi=1-\frac{1}{2 \pi} \cos ^{-1}\left(-\frac{b^{2}+2}{3 b}\right) .
$$

Note that the only real solutions of (B7) occur for $2 \geqslant|b| \geqslant 1$. Substituting (B7) in the Poincare map gives

$$
\tau=\frac{1}{2}-\frac{1}{2 \pi}\left[\cos ^{-1}\left(-\frac{b^{2}+2}{3 b}\right)+\tan ^{-1}\left(\frac{1-b^{2} / 4}{b^{2}-1}\right)^{1 / 2}\right] .
$$

This is the boundary between $1: 0$ and $2: 0$ phase locking regions for $0<\tau<\frac{1}{2}$ and $-2<b<-1$.

The boundary between the $1: 0$ and $1: 1$ phase locking zones for $b<-2$ is associated with a change in rotation number. Assuming that there is a fixed point $\phi^{*}=0$ on the Poincare map (11) we find that

$$
\tau=\frac{1}{2} \text {. }
$$

The intersection of (B8) and (B9) gives the singular point on the common boundaries of the $1: 0,1: 1$, $2: 2,2: 1$ and $2: 0$ phase locking regions. The intersection occurs for $\tau=\frac{1}{2},|b|=2$. 
The boundary between $2: 0$ and $2: 1$ phase locking occurs when

$$
T^{2}(0)=0 .
$$

Substituting in the Poincaré map (11) we find

$$
\tau=\frac{1}{2}-\frac{1}{2 \pi} \cos ^{-1}\left(-\frac{b}{2}\right)=\frac{1}{2 \pi} \cos ^{-1} \frac{b}{2} .
$$

Note that the fixed points along the boundary in (B11) occur for $\phi=0, \phi=0.5+\tau$.

The singular point on the common boundaries of the $2: 0,2: 1,4: 2,4: 1$ and $4: 0$ phase locking regions occurs when $(\mathrm{B} 10)$ is satisfied along with the criterion

$$
\frac{\partial T^{2}}{\partial \phi}=\left.\left.\frac{\partial T}{\partial \phi}\right|_{\phi=0} \frac{\partial T}{\partial \phi}\right|_{\phi=0.5+\tau}=-1
$$

where we have used the chain rule. Using (7) we find the singular point occurs for

$$
b=1-5^{1 / 2}, \quad \tau=\frac{1}{2 \pi} \cos ^{-1}\left(\frac{1-5^{1 / 2}}{2}\right) \simeq 0.356 .
$$

\section{References}

Arnold, V. I.: Small denominators. I. Mappings of the circumference onto itself. Trans. of the A.M.S. Series 2. 46, $213-284$ (1965)

Arnold, V. I.: Ordinary differential equations. Cambridge, Mass. : MIT Press, 1973

Ayers, J. L., Selverston, A. I. : Synaptic control of an endogenous pacemaker network. J. Physiol. (Paris) $73,454-561(1977)$

Bellet, S.: Clinical disorders of the heart beat (3rd edition). Philadelphia: Lea and Febiger, 1971

Cartwright, M. L., Littlewood, J. E.: On nonlinear differential equations of the second order: I. The equation $\ddot{y}-k\left(1-y^{2}\right) \dot{y}+y=b \lambda k \cos (\lambda t+\alpha), k$ large. J. London Math. Soc. 20, 180-189 (1945)

Chung, E. K.: Principles of cardiac arrhythmias. Baltimore: Williams and Wilkins, 1971

Crutchfield, J. P., Huberman, B. A.: Fluctuations and the onset of chaos. Phys. Lett. A77, 407-409 (1980)

Flaherty, J. E., Hoppensteadt, F. C. : Frequency entrainment of a forced van der Pol oscillator. Studies in Appl. Math. 58, 5-15 (1978)

Glass, L., Mackey, M. C. : A simple model for phase locking of biological oscillators. J. Math. Biol. 7, $339-352(1979)$

Glass, L., Graves, C., Petrillo, G. A., Mackey, M. C.: Unstable dynamics of a periodically driven oscillator in the presence of noise. J. theor. Biol. 86, 455-475 (1980)

Gollub, J. P., Romer, E. J., Socolar, J. E. : Trajectory divergence for coupled relaxation oscillators: Measurements and models. J. Stat. Phys. 23, $321-333$ (1980)

Grant, R. P.: The mechanism of A-V arrhythmias with an electronic analogue of the human A-V node. Am. J. Med. 20, $334-344$ (1956)

Guckenheimer, J.: On the bifurcation of maps of the interval. Inventiones Math. 39, 165-178 (1977)

Guckenheimer, J.: Symbolic dynamics and relaxation oscillations. Physica 1D, $227-235$ (1980)

Guevara, M. R., Glass, L., Shrier, A.: Phase locking, period doubling bifurcations, and irregular dynamics in periodically stimulated cardiac cells. Science 214, 1350-1353 (1981)

Guttman, R., Feldman, L., Jakobsson, E. : Frequency entrainment of squid axon membrane. J. Memb. Biol. 56, 9-18 (1980)

Herman, M. R. : Mesure de Lebesgue et nombre de rotation. In: Lecture notes in mathematics, no. 597 , Geometry and topology, pp. $271-293$. Berlin-Heidelberg-New York: Springer 1977

Hodgkin, A. L., Huxley, A. F.: A quantitative description of membrane current and its application to conduction and excitation in nerve. J. Physiol. (Lond.) 117, 500-544 (1952)

Holden, A. V.: The response of excitable membrane models to a cyclic input. Biological Cybernetics 21, $1-7$ (1976)

Hoppensteadt, F. C. : Electrical models of neurons. Lectures in Appl. Math. 19, 327-344 (1981) 
Huberman, B. A., Crutchfield, J. P.: Chaotic states of anharmonic systems in periodic fields. Phys. Rev. Lett. 43, $1743-1747$ (1979)

James, T. N., Isobe, J. H., Urthaler, F.: Correlative electrophysiological and anatomical studies concerning the site of origin of escape rhythm during complete atrioventricular block in the dog. Circ. Res. 45, 108 - 119 (1979)

Jalife, J., Moe, G. K.: A biologic model of parasystole. Am. J. Cardiol. 43, $761-772$ (1979)

Katholi, C. R., Urthaler, F., Macy, J. Jr., James, T. N.: A mathematical model of automaticity in the sinus node and AV junction based on weakly coupled relaxation oscillators. Comp. Biomed. Research 10, 529-543 (1977)

Keener, J. P.: Chaotic behaviour in piecewise continuous difference equations. Trans. Am. Math. Soc. 261, $589-604(1980)$

Keener, J. P.: Chaotic cardiac dynamics. Lectures in Appl. Math. 19, 299-325 (1981a)

Keener, J. P.: On cardiac arrhythmias: AV conduction block. J. Math. Biol. 12, 215-225 (1981b)

Keener, J. P., Hoppensteadt, F. C., Rinzel, J.: Integrate and fire models of nerve membrane response to oscillatory inputs. SIAM J. Appl. Math. 41, 503-517 (1981)

Knight, B. W.: Dynamics of encoding in a population of neurons. J. General Physiol. 59, 734-766 (1972)

Landahl, H. D., Griffeath, D.: A mathematical model for first degree block and the Wenckebach phenomenon. Bull. Math. Biophys. 33, 27-38 (1971)

Levi, M.: Qualitative analysis of the periodically forced relaxation oscillations. Memoirs Amer. Math. Soc. 32, Number 244 (1981)

Levinson, N.: A second order differential equation with singular solutions. Ann. Mathem. 50, 127-153 (1949)

Lewis, T., Mathison, G. C.: Auriculo-ventricular heart block as a result of asphyxia. Heart 2, 47-53 (1910)

Li, T.-Y., Yorke, J. A.: Period three implies chaos. Am. Math. Monthly 82, $985-992$ (1975)

Mandel, W. J. (ed.): Cardiac arrhythmias: Their mechanisms, diagnosis, and management. Philadelphia: J. B. Lippincott, 1980

May, R. M.: Simple mathematical models with very complicated dynamics. Nature 261, 459-467 (1976)

McAllister, R. E., Noble, D., Tsien, R. W.: Reconstruction of the electrical activity of cardiac Purkinje fibres. J. Physiol. (London) 251, 1 - 59 (1975)

Metropolis, N., Stein, M. L., Stein, P. R.: On finite limit sets for transformation on the unit interval. J. Combinat. Theor. 15, 25-44 (1973)

Moe, G. K., Jalife, J., Mueller, W. J., Moe, B.: A mathematical model of parasystole and its application to clinical arrhythmias. Circulation 56, 968-979 (1977)

Moulopoulos, S. D., Kardaras, N., Sideris, D. A.: Stimulus-response relationship in dog ventricle in vivo. Am. J. Physiol. 208, 154-157 (1965)

Nadeau, R. A., James, T. N.: The behaviour of atrio-ventricular nodal thythm following direct perfusion of the sinus node. Can. J. Physiol. Pharmacol. 44, 317-324 (1966)

Pavlidis, T.: Biological oscillators: Their mathematical analysis. New York: Academic Press 1973

Perkel, D. H., Schulman, J. H., Bullock, T. H., Moore, G. P., Segundo, J. P.: Pacemaker neurons: Effects of regularly spaced synaptic input. Science 145, 61-63 (1964)

Petrillo, G. A.: Phase locking: A dynamic approach to the study of respiration. Ph.D. Thesis. McGill University (Montreal) 1981

Pinsker, H. M.: Aplysia bursting neurons as endogenous oscillators. II. Synchronization and entrainment by pulsed inhibitory synaptic input. J. Neurophysiol. 40, 544-556 (1977)

Reid, J. V. O.: The cardiac pacemaker: Effects of regularly spaced nervous input. Am. Heart J. 78, $58-64(1969)$

Roberge, F. A., Nadeau, R. A., James, T. N.: The nature of the PR interval. Cardiovasc. Res. 2, 19-30 (1968)

Roberge, F. A., Nadeau, R. A.: The nature of Wenckebach cycles. Can. J. Physiol. Pharmacol. 42, $695-704(1969)$

Sarkovskiî, A. N.: Coexistence of cycles of a continuous map of a line into itself. Ukr. Mat. Z. 16, 61-71 (1964)

Schamroth, L.: The disorders of cardiac rhythm. Oxford: Blackwell 1971 
Scott, S. W.: Stimulation simulations of young yet cultured beating hearts. Ph.D. Thesis. S. U. N. Y. (Buffalo) 1979

Segers, M. : L'alternance du temps de conduction auriculo-ventriculaire. Arch. Mal. Coeur 44, 525 - 527 (1951)

Sideris, D. A., Moulopoulos, S. D.: Mechanism of atrioventricular conduction: Study on an analogue. J. Electrocardiol. 10, $51-58$ (1977)

Štefan, P. : A theorem of Šarkovskiǐ on the existence of periodic orbits of continuous endomorphisms of the real line. Commun. math. Phys. 54, 237-248 (1977)

Tomita, K., Kai, T.: Chaotic behavior of deterministic orbits: The problem of turbulent phase. Prog. Theor. Physics (Suppl. No. 64), 280-294 (1978)

Tsien, R. W., Siegelbaum, S.: Excitable tissue: The heart, chapter 20. In: Andreotti, T. E., Hoffman, J. F., Fanestil, D. D. (eds.), Physiology of membrane disorders. New York: Plenum 1978

Urthaler, F., Katholi, C. R., Macy, J. Jr., James, T. N.: Mathematical relationship between automaticity of the sinus node and the AV junction. Am. Heart J. 86, 189-196 (1973)

Urthaler, F., Katholi, C. R., Macy, J. Jr., James, T. N.: Electrophysiological and mathematical characteristics of the escape rhythm during complete AV block. Cardiovasc. Res. 8, 173-186 (1974)

Ushiyama, J., Brooks, C. McC.: Interaction of oscillators: Effect of sinusoidal stretching of the sinoatrial node on nodal rhythm. J. Electrocardiol. 10, 39-44 (1977)

van der Pol, B., van der Mark, J.: The heartbeat considered as a relaxation oscillation, and an electrical model of the heart. Phil. Mag. 6, 763-775 (1928)

van der Tweel, L. H., Meijler, F. L., van Capelle, F. J. L. : Synchronization of the heart. J. Appl. Physiol. 34, $283-287$ (1973)

Watanabe, T., Dreifus, L. S.: Atrioventricular block: Basic concepts. Chapter 16. In: Mandel, W. J. (ed.), Cardiac arrhythmias: Their mechanisms, diagnosis, and management. Philadelphia: J. B. Lippincott 1980

Winfree, A. T.: Resetting biological clocks. Physics Today 28, 34-39 (1975)

Winfree, A. T.: Phase control of neural pacemakers. Science 197, 761-763 (1977)

Winfree, A. T.: The geometry of biological time. New York: Springer 1980

Ypey, D. L., Von Meerwijk, W. P. M., Ince, E., Groos, G.: Mutual entrainment of two pacemaker cells. A study with an electronic parallel conductance model. J. Theor. Biol. 86, $731-755$ (1980)

Zaslavsky, G. M.: The simplest case of a strange attractor. Physics 69A, 145-147 (1978)

Received May 4/Revised September 14, 1981 